\title{
Cluster Correspondence Analysis
}

\author{
M. van de Velden * ${ }^{*} \quad$ A. Iodice D’Enza ${ }^{\dagger} \quad$ F. Palumbo ${ }^{\ddagger}$
}

Econometric Institute Report: 2014-24

\begin{abstract}
A new method is proposed that combines dimension reduction and cluster analysis for categorical data. A least-squares objective function is formulated that approximates the cluster by variables cross-tabulation. Individual observations are assigned to clusters in such a way that the distributions over the categorical variables for the different clusters are optimally separated. In a unified framework, a brief review of alternative methods is provided and performance of the methods is appraised by means of a simulation study. The results of the joint dimension reduction and clustering methods are compared with cluster analysis based on the full dimensional data. Our results show that the joint dimension reduction and clustering methods outperform, both with respect to the retrieval of the true underlying cluster structure and with respect to internal cluster validity measures, full dimensional clustering. The differences increase when more variables are involved and in the presence of noise variables.
\end{abstract}

\section{Introduction}

Cluster analysis aims to find a meaningful allocation of observations to groups that are similar with respect to a set of observed variables. Depending on the kind of data, an appropriate similarity measure is selected and used to allocate observations to clusters of points with high similarity within a cluster and small similarity between the clusters. To interpret cluster analysis solutions, the distributions over the variables in the different clusters can be considered. When many variables are involved, computation of all dissimilarities may become cumbersome. Moreover, interpretation of the results in terms of (relative) distributions of the variables may not be straightforward. Dimension reduction and visualization techniques can be used to overcome computational issues

${ }^{*}$ Econometric Institute, Erasmus University Rotterdam, P.O. Box 1738, 3000 DR Rotterdam, The Netherlands vandevelden@ese. eur.nl

${ }^{\dagger}$ Università di Cassino e del Lazio Meridionale, Italy iodicede@unicas.it

¥Università degli Studi di Napoli Federico II, fpalumbo@unina.it 
and at the same time facilitate a more straightforward interpretation of the cluster solutions. In this paper, we concern ourselves with clustering of high-dimensional categorical data. Existing dimension reduction and cluster analysis methods are reviewed, and we propose a new method that jointly yields optimally separated clusters and a low-dimensional approximation of the cluster by variable associations.

For continuous data, several proposals exist that combine dimension reduction and cluster analysis. Such combined approaches are typically used because the dimensionality of the data is such that computational problems arise. One straightforward approach is to first apply dimensionality reduction (e.g., principal component analysis) and then perform cluster analysis on the reduced space solution. This method is referred to as the tandem approach. Intuitive and straightforward as this approach may be, it may not yield optimal cluster allocations as the two involved methods optimize different criteria. For example, in principal component analysis, the objective is to find a small set of linear combinations of the variables that maximize explained variance. Cluster analysis, on the other hand, aims to find similar and dissimilar observations in the data set and allocate the observations accordingly to clusters. If the clustering of observations occurs in higher dimensions (i.e., dimensions not included in the principal component analysis solution) those clusters are missed. This problem is well-known (e.g., Vichi \& Kiers, 2001) and solutions have been proposed. In particular, De Soete \& Carroll (1994) proposed reduced K-means and Vichi \& Kiers (2001) proposed factorial K-means. Recently, Yamamoto \& Hwang (2014) as well as Vichi et al. (2009) provide a framework exposing the relationship between these methods and showing how the two can be joined into one objective. The latter paper also covers the case of mixed, that is, continuous and categorical, variables.

The potential problem of identifying non-existing clusters, or failing to identify existing clusters, in the reduced space has also been used as motivation for joint dimension reduction and clustering methods for categorical data. In particular, Van Buuren \& Heiser (1989) and Hwang et al. (2006) proposed methods that avoid potential problems associated with the tandem approach when applied to categorical data. For categorical data is it not obvious that similar problems do in fact occur. On the other hand, the specific nature of categorical data may in fact result in problems of a different kind. For example, categorical data quantification or scaling, permits visualization of the data into a metric space. This is not a trivial point: differently from interval data, scaling is the only way to visualize proximities in categorical data analysis. Furthermore, whereas, in the case of continuous data, the dimensionality of the data typically corresponds to the dimensionality of the data matrix, this is not necessarily the case for categorical data. If the 
categorical data are coded using indicator (dummy) matrices, the dimensionality of the data and the dimensionality of the data matrix do not correspond. In this paper, we study the performance of the tandem approach, joint dimension reduction and cluster analysis methods as well that of full dimensional clustering of categorical data. In addition, we introduce a new method that joins simple correspondence analysis and cluster analysis. The visualization of the obtained solution is straightforward and allows for a standard biplot interpretation.

The contribution of this paper is threefold. First, a new joined correspondence analysis and cluster analysis method yielding a visualization of the categories, the cluster means as well as the individual subject coordinates, is presented. Secondly, we provide a comprehensive overview of existing dimension reduction and clustering methods for categorical variables, and we point out that different scaling methods can lead to similar cluster solutions whilst yielding different data visualizations. Moreover, we resolve some issues concerning these methods and propose a new algorithm for GROUPALS; a method proposed by Van Buuren \& Heiser (1989). Thirdly, using a simulation study and a real data example, we appraise the performance of the joint dimension reduction and clustering methods as well as that of the tandem approach and full dimensional clustering of the categorical data. Such a comparative study of the different dimension reduction and cluster analysis methods does not exist. In a recent review by Iodice D'Enza et al. (2014), the theoretical relationships between existing methods was captured and illustrated by means of one empirical example. Similarly, Hwang et al. (2006) compared the results of their method to those obtained using the method proposed by Van Buuren \& Heiser (1989) using one empirical example. In our simulation study, however, we appraise the performance of all joint dimension reduction and cluster methods, as well as the tandem approach and full dimensional clustering of the categorical data, under various, realistic, conditions.

The remainder of this paper is organized as follows. In Section 2, notation and some essential correspondence analysis formulas are given. Then, in Section 3, the new method is presented. In Section 4.1, we derive a new algorithm for GROUPALS based on the first-order conditions corresponding to the original problem. Hwang et al. (2006)'s method is shortly presented in Section 4.2 followed by a brief summary of Iodice D'Enza \& Palumbo (2013)'s approach. In Section 5, the performance of all methods is assessed by means of a simulation study based on categorical data generated according to different underlying profiles for the different clusters of individuals. We illustrate the new method by means of a real data set on the preferences of different humor styles in Section 6. We summarize our findings in Section 7. 


\section{Correspondence analysis}

Correspondence analysis has been invented and reinvented several times (see, e.g., Nishisato, 1980; Greenacre, 1984) for an historical overview of the method). As a consequence, the method can be derived and presented in many ways. Here we do not concern ourselves with these issues and alternate between different rationales. In particular, without providing details on their origins and interpretations, we use different formulations and properties to simplify our exposition of the new method.

Let $\mathbf{P}$ denote a $q_{r} \times q_{c}$ data matrix with nonnegative elements that sum to 1 . That is, $\mathbf{1}_{q_{r}}^{\prime} \mathbf{P} \mathbf{1}_{q_{c}}=$ 1, where, generically, $\mathbf{1}_{q}$ denotes a $q$ dimensional vector of ones. Correspondence analysis amounts to the following least-squares approximation problem:

$$
\min _{\mathbf{A}, \mathbf{B}}\left\|\tilde{\mathbf{P}}-\mathbf{D}_{r}^{1 / 2} \mathbf{A} \mathbf{B}^{\prime} \mathbf{D}_{c}^{1 / 2}\right\|^{2}
$$

where $\left.\tilde{\mathbf{P}}=\mathbf{D}_{r}^{-1 / 2}(\mathbf{P}-\mathbf{r c})^{\prime}\right) \mathbf{D}_{c}^{-1 / 2}, \mathbf{r}=\mathbf{P} \mathbf{1}_{q_{c}}, \mathbf{c}=\mathbf{P}^{\prime} \mathbf{1}_{q_{r}}, \mathbf{D}_{r}$ and $\mathbf{D}_{c}$ are corresponding diagonal matrices (i.e., $\mathbf{D}_{r} \mathbf{1}_{q_{r}}=\mathbf{r}$ and $\mathbf{D}_{c} \mathbf{1}_{q_{c}}=\mathbf{c}$ ). The so-called row and column coordinate matrices $\mathbf{A}$ and $\mathbf{B}$ are of rank $k$, where $k$ is the dimensionality of the approximation. By imposing

$$
\mathbf{B}^{\prime} \mathbf{D}_{c} \mathbf{B}=\mathbf{I}_{k}
$$

a solution can be obtained by using the singular value decomposition

$$
\tilde{\mathbf{P}}=\mathbf{U} \boldsymbol{\Lambda} \mathbf{V}^{\prime}
$$

where $\mathbf{U}$ and $\mathbf{V}$ are orthonormal and $\boldsymbol{\Lambda}$ is a diagonal matrix with, in descending order, the singular values on its diagonal. By selecting only the first $k$ columns of $\mathbf{U}$ and $\mathbf{V}$ and the corresponding singular values, a $k$-dimensional least-squares approximation of $\tilde{\mathbf{P}}$ is obtained. The resulting coordinate matrices are

$$
\mathbf{A}=\mathbf{D}_{r}^{-1 / 2} \mathbf{U} \boldsymbol{\Lambda} \text { and } \mathbf{B}=\mathbf{D}_{c}^{-1 / 2} \mathbf{V}
$$

so that

$$
\mathbf{A}^{\prime} \mathbf{D}_{r} \mathbf{A}=\Lambda^{2}
$$

In this formulation, the row-coordinates are referred to as principal coordinates whereas the column coordinates are standard coordinates. This set of coordinates consitutes a so-called biplot (see, 
e.g., Gower \& Hand, 1996) as the inner-product $\mathbf{D}_{r}^{1 / 2} \mathbf{A} \mathbf{B}^{\prime} \mathbf{D}_{c}^{1 / 2}$ approximates the data.

If $\mathbf{P}$ is obtained from a contingency table, the matrix $\tilde{\mathbf{P}}$ is the matrix of standardized residuals (i.e., the matrix of standardized deviations from the independence model). The biplot coordinates collected in $\mathbf{A}$ and $\mathbf{B}$ give a low-dimensional approximation of these standardized residuals.

It is easily verified that the minimization problem (1) is equivalent to maximizing the sum of squared singular values. That is:

$$
\max \operatorname{trace} \boldsymbol{\Lambda}^{2}=\max \operatorname{trace} \mathbf{A}^{\prime} \mathbf{D}_{r} \mathbf{A},=\max \left\|\mathbf{D}_{r}^{1 / 2} \mathbf{A}\right\|^{2}
$$

subject to

$$
\mathbf{B}^{\prime} \mathbf{D}_{c} \mathbf{B}=\mathbf{I}_{k} .
$$

This formulation will prove useful in our later expositions. Note that, from (2) it follows that the correspondence coordinates can be interpreted as optimal scaling values that, when used as weights for rows and columns, maximize the variance between rows (columns) whilst minimizing the variance within a row (column). For a complete exposition of CA derived in this fashion see, Nishisato (1994).

\subsection{Correspondence analysis of more than two categorical variables}

For the analysis of more than two variables, several extensions of correspondence analysis exist. Most extensions amount to applying correspondence analysis to a particularly formatted data matrix. Let $\mathbf{Z}_{j}$ denote an $n \times q_{j}$ indicator matrix. That is, each row corresponds to an observation, and the columns represent categories. Observed categories are coded by ones and all other elements are zero. Consequently, $\mathbf{Z}_{j} \mathbf{1}_{q_{j}}=\mathbf{1}_{n}$. Data on several categorical variables can be collected in a so-called superindicator matrix $\mathbf{Z}=\left[\mathbf{Z}_{1}, \ldots, \mathbf{Z}_{p}\right]$. The most popular extension, multiple correspondence analysis (MCA), amounts to either applying correspondence analysis to the superindicator matrix $\mathbf{Z}$ or to the so-called Burt matrix, that is, the collection of all two-way cross-tabulations calculated by: $\mathbf{B}=\mathbf{Z}^{\prime} \mathbf{Z}$.

Another approach, particularly appropriate when there is reason for an asymmetric treatment of the categorical variables, concerns the analysis of all cross-tabulations of one (set of) categorical variable(s) with all other categorical variables. In this setting, the cross-tabulations are gathered in a concatenated table which is subjected to correspondence analysis. Note that, in this way, not all interactions are coded (and approximated) as the concatenated table represents the association 
of one (set of) categorical variable(s) with all other categorical variables. It is this extension of $\mathrm{CA}$ that we use in our cluster correspondence analysis approach.

\section{Cluster correspondence analysis}

Assume we have data of $n$ individuals on $p$ categorical variables gathered in a super indicator matrix $\mathbf{Z}$ of dimensionality $n \times Q$, where $Q=\sum_{j=1}^{p} q_{j}$. We are interested in finding $K$ clusters of the $n$ individuals based on the observations on the categorical variables. Cluster membership itself can also be considered as a categorical variable and this can be coded using an indicator matrix, say $\mathbf{Z}_{K}$. To consider the association of the clusters with the categorical variables, we can construct a table cross-tabulating cluster memberships with the categorical variables as $\mathbf{F}=\mathbf{Z}_{K}^{\prime} \mathbf{Z}$, where $\mathbf{Z}_{K}$ is the $n \times K$ indicator matrix indicating cluster membership. Applying CA to this matrix yields optimal scaling values for rows (clusters) and columns (categories) in such a way that the between cluster variance is a maximum. That is, the clusters are optimally separated with respect to the distributions over the categorical variables.

Using the definitions introduced in the previous section, we let

$$
\mathbf{P}=\frac{1}{n p} \mathbf{F}
$$

so that for $\mathbf{P}-\mathbf{r c}^{\prime}$ we get

$$
\mathbf{P}-\mathbf{P} \mathbf{1 1} \mathbf{1}^{\prime} \mathbf{P}=\frac{1}{n p}\left(\mathbf{F}-\frac{1}{n p} \mathbf{F} \mathbf{1}_{n} \mathbf{1}_{Q}^{\prime} \mathbf{F}\right)=\frac{1}{n p}\left(\mathbf{Z}_{K}^{\prime} \mathbf{Z}-\frac{1}{n} \mathbf{Z}_{K}^{\prime} \mathbf{1}_{n} \mathbf{1}_{n}^{\prime} \mathbf{Z}\right)=\frac{1}{n p} \mathbf{Z}_{K}^{\prime} \mathbf{M Z},
$$

where $\mathbf{M}=\mathbf{I}_{n}-\mathbf{1}_{n} \mathbf{1}_{n}^{\prime} / n$. Furthermore, define a diagonal matrix $\mathbf{D}_{z}$ so that $\mathbf{D}_{z} \mathbf{1}=\mathbf{Z}^{\prime} \mathbf{1}$ and let $\mathbf{D}_{K}=\mathbf{Z}_{K}^{\prime} \mathbf{Z}_{K}$, a diagonal matrix with cluster sizes. The correspondence analysis objective function (1) for the cluster by variable case, becomes

$$
\min _{\mathbf{z}_{K}, \mathbf{G}, \mathbf{B}}\left\|\frac{1}{\sqrt{p}} \mathbf{D}_{K}^{-1 / 2} \mathbf{Z}_{K}^{\prime} \mathbf{M} \mathbf{Z} \mathbf{D}_{z}^{-1 / 2}-\frac{1}{n \sqrt{p}} \mathbf{D}_{K}^{1 / 2} \mathbf{G B}^{\prime} \mathbf{D}_{z}^{1 / 2}\right\|^{2}
$$

Upon defining

$$
\mathbf{G}^{*}=\frac{1}{\sqrt{n}} \mathbf{D}_{K}^{1 / 2} \mathbf{G} \text { and } \mathbf{B}^{*}=\frac{1}{\sqrt{n p}} \mathbf{D}_{z}^{1 / 2} \mathbf{B}
$$


we can re-express (3) as

$$
\min _{\mathbf{Z}_{K}, \mathbf{G}^{*}, \mathbf{B}^{*}}\left\|\frac{1}{\sqrt{p}} \mathbf{D}_{K}^{-1 / 2} \mathbf{Z}_{K}^{\prime} \mathbf{M} \mathbf{Z} \mathbf{D}_{z}^{-1 / 2}-\mathbf{G}^{*} \mathbf{B}^{*}\right\|^{2}
$$

This objective function is minimized subject to $\mathbf{B}^{*{ }^{\prime}} \mathbf{B}^{*}=\mathbf{I}_{k}$.

To solve this problem, we first consider $\mathbf{Z}_{K}$ to be known and minimize with respect to $\mathbf{G}^{*}$ and $\mathbf{B}^{*}$. This is a standard matrix approximation problem. The solution can be obtained directly from the singular value decomposition

$$
\frac{1}{\sqrt{p}} \mathbf{D}_{K}^{-1 / 2} \mathbf{Z}_{K}^{\prime} \mathbf{M Z D}_{z}^{-1 / 2}=\mathbf{U} \mathbf{\Lambda} \mathbf{V}^{\prime}
$$

and by letting

$$
\mathbf{B}^{*}=\mathbf{V} \text { and } \mathbf{G}^{*}=\mathbf{U} \boldsymbol{\Lambda}
$$

The appropriately scaled solution for the rows (i.e., the clusters) and columns (i.e., the categories) thus becomes

$$
\mathbf{B}=\sqrt{n q} \mathbf{D}_{z}^{-1 / 2} \mathbf{V} \text { and } \mathbf{G}=\sqrt{n} \mathbf{D}_{K}^{-1 / 2} \mathbf{G}^{*}
$$

In addition to the low-dimensional matrix approximation involving $\mathbf{B}$ and $\mathbf{G}$, we need to determine the optimal cluster allocation $\mathbf{Z}_{K}$. That is, $\mathbf{Z}_{K}$ must be determined in such a way that (1) is a minimum. As $\mathbf{Z}_{K}$ is an indicator matrix this is not a trivial problem. However, recall that the CA objective function (1) is equivalent to the optimal scaling objective function (2). Hence, (3) coincides with

$$
\max \left\|\frac{1}{\sqrt{n}} \mathbf{D}_{K}^{1 / 2} \mathbf{G}\right\|^{2}=\max \operatorname{trace}\left(\mathbf{G}^{\prime} \mathbf{D}_{r} \mathbf{G}\right)=\max \operatorname{trace} \boldsymbol{\Lambda}^{2}
$$

subject to

$$
\mathbf{B}^{\prime} \mathbf{D}_{c} \mathbf{B}=\mathbf{I}_{k}
$$

Now, from (5), (6) and (7), it follows that

$$
\mathbf{G}=\sqrt{\frac{n}{p}} \mathbf{D}_{K}^{-1} \mathbf{Z}_{K}^{\prime} \mathbf{M Z D}_{z}^{-\frac{1}{2}} \mathbf{V}
$$

so that, for fixed $\mathbf{V}$, objective (8), which is equivalent to (3), can be expressed as

$$
\max _{\mathbf{Z}_{K}} \phi=\left\|\frac{1}{\sqrt{p}} \mathbf{D}_{K}^{-1 / 2} \mathbf{Z}_{K}^{\prime} \mathbf{M} \mathbf{Z} \mathbf{D}_{z}^{-\frac{1}{2}} \mathbf{V}\right\|^{2}
$$


This optimization problem is in fact equivalent to a K-means clustering problem. That is, maximizing $\phi$ with respect to $\mathbf{Z}_{K}$, is equivalent to solving the following $\mathrm{K}$-means objective:

$$
\min _{\mathbf{Z}_{K}, \mathbf{G}} \phi^{\prime}=\left\|\frac{1}{\sqrt{p}} \mathbf{M} \mathbf{Z} \mathbf{D}_{z}^{-\frac{1}{2}} \mathbf{V}-\mathbf{Z}_{K}^{\prime} \mathbf{G}\right\|^{2}
$$

Proof. First of all, note that

$$
\phi=\left\|\frac{1}{\sqrt{p}} \mathbf{D}_{K}^{-1 / 2} \mathbf{Z}_{K}^{\prime} \mathbf{M} \mathbf{Z} \mathbf{D}_{z}^{-\frac{1}{2}} \mathbf{V}\right\|^{2}=\operatorname{trace} \frac{1}{p} \mathbf{V}^{\prime} \mathbf{D}_{z}^{-\frac{1}{2}} \mathbf{Z}^{\prime} \mathbf{M} \mathbf{Z}_{K} \mathbf{D}_{K}^{-1} \mathbf{Z}_{K}^{\prime} \mathbf{M} \mathbf{Z} \mathbf{D}_{z}^{-\frac{1}{2}} \mathbf{V} .
$$

Next, let

$$
\mathbf{Y}=\sqrt{\frac{n}{p}} \mathbf{M Z D}_{z}^{-\frac{1}{2}} \mathbf{V}
$$

and rewrite the K-means objective (10), as

$$
\min _{\mathbf{Z}_{K}, \mathbf{G}} \phi^{\prime}=\left\|\mathbf{Y}-\mathbf{Z}_{K} \mathbf{G}\right\|^{2}
$$

Solving this K-means problem with respect to $\mathbf{G}$ yields

$$
\mathbf{G}=\left(\mathbf{Z}_{K}^{\prime} \mathbf{Z}_{K}\right)^{-1} \mathbf{Z}_{K}^{\prime} \mathbf{Y}=\mathbf{D}_{K}^{-1} \mathbf{Z}_{K}^{\prime} \mathbf{Y}
$$

which is in accordance with (9). Inserting this into the K-means objective we get

$$
\begin{aligned}
\min _{\mathbf{Z}_{K}, \mathbf{G}}\left\|\mathbf{Y}-\mathbf{Z}_{K} \mathbf{G}\right\|^{2} & =\operatorname{trace} \mathbf{Y}^{\prime} \mathbf{Y}+\operatorname{trace} \mathbf{G}^{\prime} \mathbf{D}_{K} \mathbf{G}-2 \operatorname{trace} \mathbf{G}^{\prime} \mathbf{Z}_{K}^{\prime} \mathbf{Y} \\
& =\operatorname{trace} \mathbf{Y}^{\prime} \mathbf{Y}+\operatorname{trace} \mathbf{Y}^{\prime} \mathbf{Z}_{K} \mathbf{D}_{K}^{-1} \mathbf{D}_{K} \mathbf{D}_{K}^{1} \mathbf{Z}_{K}{ }^{\prime} \mathbf{Y}-2 \operatorname{trace} \mathbf{Y}^{\prime} \mathbf{Z}_{K} \mathbf{D}_{K}^{-1} \mathbf{Z}_{K}{ }^{\prime} \mathbf{Y} \\
& =\operatorname{trace} \mathbf{Y}^{\prime} \mathbf{Y}-\operatorname{trace} \mathbf{Y}^{\prime} \mathbf{Z}_{K} \mathbf{D}_{K}^{-1} \mathbf{Z}_{K}{ }^{\prime} \mathbf{Y} .
\end{aligned}
$$

So, minimizing the K-means objective amounts to maximizing

$$
\operatorname{trace} \mathbf{Y}^{\prime} \mathbf{Z}_{K} \mathbf{D}_{K}^{-1} \mathbf{Z}_{K}{ }^{\prime} \mathbf{Y}=n \operatorname{trace} \frac{1}{p} \mathbf{V}^{\prime} \mathbf{D}_{z}^{-\frac{1}{2}} \mathbf{Z}^{\prime} \mathbf{M} \mathbf{Z}_{K} \mathbf{D}_{K}^{-1} \mathbf{Z}_{K}^{\prime} \mathbf{M Z D _ { z } ^ { - \frac { 1 } { 2 } }} \mathbf{V}
$$

We see that (11) and (13) are equivalent. Hence, for fixed $\mathbf{V}$, we can find a cluster allocation $\mathbf{Z}_{K}$ by applying the K-means algorithm to $\mathbf{Y}$. The resulting cluster allocation $\mathbf{Z}_{K}$ yields an improved (i.e., increased) value for the objective function. Using the new $\mathbf{Z}_{K}$, we repeat the CA step to update the optimal scaling values for the rows and columns. 
The resulting algorithm for cluster correspondence analysis can be summarized as follows:

1. Generate an initial cluster allocation $\mathbf{Z}_{K}$ (e.g., by randomly assigning subjects to clusters).

2. Find cluster and category quantifications $\mathbf{G}$ and $\mathbf{B}$ using (7).

3. Use (12) to construct an initial configuration for the subjects $\mathbf{Y}$.

4. Find updates for $\mathbf{Z}_{K}$ (and $\mathbf{G}$ ) by applying $\mathrm{K}$-means clustering to $\mathbf{Y}$ (using $\mathbf{G}$ as initial matrix of cluster means).

5. Repeat the procedure (i.e. go back to step 2) using $\mathbf{Z}_{K}$ for the cluster allocation matrix, until convergence. That is, until $\mathbf{Z}_{K}$ (and hence $\mathbf{Y}$ and $\mathbf{G}$ ) remain constant.

Note that, convergence is guaranteed as the value of the objective function (8) never decreases in subsequent steps. Obviously, there is no guarantee that the obtained optimum is global. Random starts can be used to reduce the chances of finding a local optimum.

The new cluster correspondence analysis method can be seen as a correspondence analysis of cross-tabulations of cluster memberships by categorical variables. The rows of the data matrix represent clusters and the obtained row coordinates maximize the between cluster variance. From (3), it is clear that the solution for rows and columns constitutes a biplot of cluster means and attributes. Hence, projections of cluster points on attribute vertices provide approximations to the cluster by attribute associations. The typical CA normalizations do not necessarily lead to similar spread in the row and column points. Consequently, a joint display of the row and column points is not very informative. This can be repaired without damaging the biplot property by multiplying the coordinates of one set by a constant and the other set by the inverse of that constant. In the context of biplots some proposals exist to deal with such problems (see, e.g., Gower et al., 2010, 2011). Here, we propose to use a constant $\gamma$ in such a way that the average squared deviation from the origin is the same in both sets of points. That is, define

$$
\mathbf{G}_{s}=\gamma \mathbf{G} \text { and } \mathbf{B}_{s}=\frac{1}{\gamma} \mathbf{B},
$$

where

$$
\gamma=\left(\frac{K}{Q} \operatorname{trace} \mathbf{B}^{\prime} \mathbf{B} / \operatorname{trace} \mathbf{G}^{\prime} \mathbf{G}\right)^{1 / 4},
$$

so that,

$$
\frac{1}{K} \operatorname{trace} \mathbf{G}_{s}{ }^{\prime} \mathbf{G}_{s}=\frac{1}{Q} \operatorname{trace} \mathbf{B}_{s}{ }^{\prime} \mathbf{B}_{s} .
$$


Plotting these rescaled coordinate matrices rather than the original $\mathbf{G}$ and $\mathbf{B}$, facilitates a directly interpretable visualization of the cluster by attribute associations.

\section{Related methods}

Cluster correspondence analysis combines dimension reduction with cluster analysis for categorical data. Other methods exist for such analyses. In particular, GROUPALS (Van Buuren \& Heiser, 1989), MCA K-means (Hwang et al., 2006) and iterative factorial clustering of binary variables (i-FCB; Iodice D'Enza \& Palumbo, 2013) all have similar objectives. It is therefore important to compare the new method with the existing methods both theoretically and empirically. For the three existing methods, Iodice D'Enza et al. (2014), exposed some theoretical relationships and illustrated the differences using one empirical example. To see how the new method relates to the existing ones, we briefly revisit the existing methods. Moreover, we derive a new algorithm for GROUPALS based on the first order conditions corresponding to the problem. The existing algorithm, proposed by Van Buuren \& Heiser (1989) is an alternating least-squares algorithm based on a "transformation of normalization procedure".

\subsection{GROUPALS}

Van Buuren \& Heiser (1989) formulate as objective function for GROUPALS

$$
\min _{\mathbf{B}, \mathbf{Z}_{\mathbf{K}}, \mathbf{G}} \frac{1}{p} \sum_{j=1}^{p}\left\|\mathbf{Z}_{K} \mathbf{G}-\mathbf{Z}_{j} \mathbf{B}_{j}\right\|^{2},
$$

subject to

$$
\sum_{j=1}^{q} \mathbf{B}_{j}^{\prime} \mathbf{Z}_{j}^{\prime} \mathbf{Z}_{j} \mathbf{B}_{j}=\mathbf{I}_{k}
$$

To find the first-order conditions we first fix $\mathbf{Z}_{K}$ and solve for $\mathbf{B}_{j}$ and $\mathbf{G}$ by setting up the Lagrangean:

$$
\begin{aligned}
\psi & =\frac{1}{p} \sum_{j=1}^{p} \operatorname{trace}\left(\mathbf{Z}_{K} \mathbf{G}-\mathbf{Z}_{j} \mathbf{B}_{j}\right)^{\prime}\left(\mathbf{Z}_{K} \mathbf{G}-\mathbf{Z}_{j} \mathbf{B}_{j}\right)+\operatorname{trace} \mathbf{L}\left(\sum_{j=1}^{p} \mathbf{B}_{j}^{\prime} \mathbf{D}_{j} \mathbf{B}_{j}-\mathbf{I}_{k}\right) \\
& =\operatorname{trace} \mathbf{G}^{\prime} \mathbf{Z}_{K}^{\prime} \mathbf{Z}_{K} \mathbf{G}+\frac{1}{p} \sum_{j=1}^{p} \operatorname{trace} \mathbf{B}_{j}^{\prime} \mathbf{Z}_{j}^{\prime} \mathbf{Z}_{j} \mathbf{B}_{j}-\frac{2}{p} \sum_{j=1}^{p} \operatorname{trace} \mathbf{G}^{\prime} \mathbf{Z}_{K}{ }^{\prime} \mathbf{Z}_{j} \mathbf{B}_{j}+\operatorname{trace} \mathbf{L}\left(\sum_{j=1}^{p} \mathbf{B}_{j}^{\prime} \mathbf{D}_{j} \mathbf{B}_{j}-\mathbf{I}_{k}\right) \\
& =\operatorname{trace} \mathbf{G}^{\prime} \mathbf{Z}_{K}{ }^{\prime} \mathbf{Z}_{K} \mathbf{G}+\frac{1}{p}-\frac{2}{p} \sum_{j=1}^{p} \operatorname{trace} \mathbf{G}^{\prime} \mathbf{Z}_{K}{ }^{\prime} \mathbf{Z}_{j} \mathbf{B}_{j}+\operatorname{trace} \mathbf{L}\left(\sum_{j=1}^{p} \mathbf{B}_{j}^{\prime} \mathbf{D}_{j} \mathbf{B}_{j}-\mathbf{I}_{k}\right)
\end{aligned}
$$


where $\mathbf{L}$ is the matrix of Lagrange multipliers. Taking derivatives and equating to zero yields the first order conditions.

For $\mathbf{G}$ :

$$
\begin{aligned}
2 \operatorname{trace} \mathbf{G}^{\prime} \mathbf{Z}_{K}{ }^{\prime} \mathbf{Z}_{K} d \mathbf{G} & =\frac{2}{p} \sum_{j=1}^{p} \operatorname{trace} \mathbf{B}_{j}^{\prime} \mathbf{Z}_{j}^{\prime} \mathbf{Z}_{K} d \mathbf{G} \\
\mathbf{G}^{\prime} \mathbf{Z}_{K} \mathbf{Z}_{K} & =\frac{1}{p} \sum_{j=1}^{p} \mathbf{B}_{j}^{\prime} \mathbf{Z}_{j}^{\prime} \mathbf{Z}_{K} \\
\mathbf{G} & =\frac{1}{p}\left(\mathbf{Z}_{K}{ }^{\prime} \mathbf{Z}_{K}\right)^{-1} \mathbf{Z}_{K}{ }^{\prime} \sum_{j=1}^{p} \mathbf{Z}_{j} \mathbf{B}_{j} .
\end{aligned}
$$

For $\mathbf{B}_{j}$ :

$$
\begin{aligned}
\frac{2}{p} \operatorname{trace} \mathbf{G}^{\prime} \mathbf{Z}_{K}{ }^{\prime} \mathbf{Z}_{j} d \mathbf{B}_{j} & =2 \operatorname{trace} \mathbf{L} \mathbf{B}_{j}^{\prime} \mathbf{D}_{j} d \mathbf{B}_{j} \\
\frac{1}{p} \mathbf{Z}_{j}^{\prime} \mathbf{Z}_{K} \mathbf{G} & =\mathbf{D}_{j} \mathbf{B}_{j} \mathbf{L} .
\end{aligned}
$$

Inserting the solution for $\mathbf{G}$ we obtain

$$
\frac{1}{p^{2}} \mathbf{Z}_{j}^{\prime} \mathbf{Z}_{K}\left(\mathbf{Z}_{K}{ }^{\prime} \mathbf{Z}_{K}\right)^{-1} \mathbf{Z}_{K}{ }^{\prime} \sum_{j=1}^{p} \mathbf{Z}_{j} \mathbf{B}_{j}=\mathbf{D}_{j} \mathbf{B}_{j} \mathbf{L}
$$

Note that, as the constraints are symmetric, $\mathbf{L}$ is also symmetric. Furthermore, as $j=1, \ldots, p$, we have $p$ equations. However, defining $\mathbf{Z}=\left[\mathbf{Z}_{1}, \ldots, \mathbf{Z}_{p}\right]$ and $\mathbf{B}=\left[\mathbf{B}_{1}^{\prime}, \ldots, \mathbf{B}_{p}^{\prime}\right]^{\prime}$, the $p$ equations can be expressed as

$$
\frac{1}{p^{2}} \mathbf{Z}^{\prime} \mathbf{Z}_{K}\left(\mathbf{Z}_{K}{ }^{\prime} \mathbf{Z}_{K}\right)^{-1} \mathbf{Z}_{K}{ }^{\prime} \mathbf{Z B}=\mathbf{D B L}
$$

where $\mathbf{D}$ is a block-diagonal matrix with as diagonal blocks $\mathbf{D}_{1}, \ldots, \mathbf{D}_{p}$. Premultiplying both sides by $\mathbf{D}^{-1 / 2}$ we get

$$
\frac{1}{p^{2}} \mathbf{D}^{-1 / 2} \mathbf{Z}^{\prime} \mathbf{Z}_{K}\left(\mathbf{Z}_{K}{ }^{\prime} \mathbf{Z}_{K}\right)^{-1} \mathbf{Z}_{K}{ }^{\prime} \mathbf{Z D}^{-1 / 2} \mathbf{D}^{1 / 2} \mathbf{B}=\mathbf{D}^{1 / 2} \mathbf{B L}
$$

Without loss of generality we can replace $\mathbf{L}$ by its eigendecomposition to get

$$
\frac{1}{p^{2}} \mathbf{D}^{-1 / 2} \mathbf{Z}^{\prime} \mathbf{Z}_{K}\left(\mathbf{Z}_{K}^{\prime} \mathbf{Z}_{K}\right)^{-1} \mathbf{Z}_{K}^{\prime} \mathbf{Z D}^{-1 / 2} \mathbf{D}^{1 / 2} \mathbf{B}=\mathbf{D}^{1 / 2} \mathbf{B} \mathbf{U} \boldsymbol{\Lambda} \mathbf{U}^{\prime}
$$


so that

$$
\frac{1}{p^{2}} \mathbf{D}^{-1 / 2} \mathbf{Z}^{\prime} \mathbf{Z}_{K}\left(\mathbf{Z}_{K}{ }^{\prime} \mathbf{Z}_{K}\right)^{-1} \mathbf{Z}_{K}^{\prime} \mathbf{Z D}^{-1 / 2} \mathbf{D}^{1 / 2} \mathbf{B U}=\mathbf{D}^{1 / 2} \mathbf{B U} \boldsymbol{\Lambda} .
$$

Hence, letting

$$
\mathbf{B}^{*}=\mathbf{D}^{1 / 2} \mathbf{B U}
$$

we see that $\mathbf{B}^{*}$ can be obtained by taking the first $k$ orthonormal eigenvectors (corresponding to the $k$ largest eigenvalues) of

$$
\frac{1}{p^{2}} \mathbf{D}^{-1 / 2} \mathbf{Z}^{\prime} \mathbf{Z}_{K}\left(\mathbf{Z}_{K}{ }^{\prime} \mathbf{Z}_{K}\right)^{-1} \mathbf{Z}_{K}{ }^{\prime} \mathbf{Z D}^{-1 / 2}
$$

The appropriately standardized category quantifications become

$$
\mathbf{B}=\mathbf{D}^{-1 / 2} \mathbf{B}^{*}
$$

and $\mathbf{G}$ is obtained by inserting this into the first order condition for $\mathbf{G}$, that is,

$$
\mathbf{G}=\frac{1}{p}\left(\mathbf{Z}_{K}{ }^{\prime} \mathbf{Z}_{K}\right)^{-1} \mathbf{Z}_{K}{ }^{\prime} \mathbf{Z B}
$$

To find $\mathbf{Z}_{K}$, recall the original objective function:

$$
\min _{\mathbf{B}, \mathbf{Z}_{\mathbf{K}}, \mathbf{G}} \frac{1}{p} \sum_{j=1}^{p}\left\|\mathbf{Z}_{K} \mathbf{G}-\mathbf{Z}_{j} \mathbf{B}_{j}\right\|^{2}
$$

For fixed $\mathbf{B}_{j}$,this is equivalent to considering

$$
\min _{\mathbf{B}, \mathbf{Z}_{\mathbf{K}}, \mathbf{G}}\left\|\frac{1}{p} \sum_{j=1}^{p} \mathbf{Z}_{j} \mathbf{B}_{j}-\mathbf{Z}_{K} \mathbf{G}\right\|^{2}
$$

Proof.

$$
\frac{1}{p} \sum_{j=1}^{p}\left\|\mathbf{Z}_{K} \mathbf{G}-\mathbf{Z}_{j} \mathbf{B}_{j}\right\|^{2}=\operatorname{trace} \mathbf{G}^{\prime} \mathbf{Z}_{K}{ }^{\prime} \mathbf{Z}_{K} \mathbf{G}+\frac{1}{p} \sum_{j=1}^{p} \operatorname{trace} \mathbf{B}_{j}^{\prime} \mathbf{Z}_{j}^{\prime} \mathbf{Z}_{j} \mathbf{B}_{j}-\frac{2}{p} \sum_{j=1}^{p} \operatorname{trace} \mathbf{G}^{\prime} \mathbf{Z}_{K}{ }^{\prime} \mathbf{Z}_{j} \mathbf{B}_{j},
$$

and

$$
\left\|\frac{1}{p} \sum_{j=1}^{p} \mathbf{Z}_{j} \mathbf{B}_{j}-\mathbf{Z}_{K} \mathbf{G}\right\|^{2}=\operatorname{trace} \mathbf{G}^{\prime} \mathbf{Z}_{K}{ }^{\prime} \mathbf{Z}_{K} \mathbf{G}+\frac{1}{p^{2}} \operatorname{trace} \mathbf{B}^{\prime} \mathbf{Z}^{\prime} \mathbf{Z B}-\frac{2}{p} \sum_{j=1}^{p} \operatorname{trace} \mathbf{G}^{\prime} \mathbf{Z}_{K}{ }^{\prime} \mathbf{Z}_{j} \mathbf{B}_{j} .
$$


Hence, to find $\mathbf{Z}_{K}$ we can apply K-means to the "average configuration": $\frac{1}{p} \sum_{j=1}^{p} \mathbf{Z}_{j} \mathbf{B}_{j}$.

Note: It can easily be verified that $\mathbf{D}^{1 / 2} \mathbf{1}$ is an eigenvector of (15) corresponding to the eigenvalue 1. Hence, as in $\mathrm{CA}$ and MCA, there is a so-called trivial first solution. Discarding this solution can be achieved by centering $\mathbf{Z}$. We can summarize the new GROUPALS algorithm as follows:

1. Generate an initial cluster allocation $\mathbf{Z}_{K}$ (e.g. by randomly assigning subjects to clusters).

2. Use (15), (16) and (17) to obtain $\mathbf{B}$ and $\mathbf{G}$.

3. Apply the K-means algorithm to the average configuration $\frac{1}{p} \sum_{j=1}^{p} \mathbf{Z}_{j} \mathbf{B}_{j}$, using $\mathbf{G}$ for the initial cluster means, to update $\mathbf{Z}_{K}$ and $\mathbf{G}$.

4. Return to step 2 and repeat until convergence.

\subsection{MCA K-means}

Hwang et al. (2006) propose a joined multiple correspondence analysis and K-means method that combines the two objectives using a convex combination. The objective can be formulated as follows:

$$
\min _{\mathbf{Y}, \mathbf{B}_{j}, \mathbf{G}, \mathbf{Z}_{K}} \alpha \frac{1}{p} \sum_{j=1}^{p}\left\|\mathbf{Y}-\mathbf{Z}_{j} \mathbf{B}_{j}\right\|^{2}+(1-\alpha)\left\|\mathbf{Y}-\mathbf{Z}_{K} \mathbf{G}\right\|^{2}
$$

subject to

$$
\mathbf{Y}^{\prime} \mathbf{Y}=\mathbf{I}_{k} .
$$

The weight $\alpha$ is user supplied and controls the importance of the MCA and K-means part. Note that the term $1 / p$ does not appear in Hwang et al. (2006). We have added it here to maintain the relationship with MCA. This scaling factor ensures that, for $\alpha=.5$, the MCA and cluster analysis parts receive equal weights.

It is not difficult to show that (18) can be solved by

$$
\mathbf{B}_{j}=\left(\mathbf{Z}_{j}^{\prime} \mathbf{Z}_{j}\right)^{-1} \mathbf{Z}_{j}^{\prime} \mathbf{Y} \text { and } \mathbf{G}=\left(\mathbf{z}_{K}^{\prime} \mathbf{Z}_{K}\right)^{-1} \mathbf{Z}_{K}^{\prime} \mathbf{Y}
$$

and

$$
\left(\alpha \frac{1}{p} \sum_{j=1}^{p} \mathbf{Z}_{j}\left(\mathbf{z}_{j}^{\prime} \mathbf{Z}_{j}\right)^{-1} \mathbf{Z}_{j}^{\prime}+(1-\alpha) \mathbf{Z}_{K}\left(\mathbf{Z}_{K}^{\prime} \mathbf{Z}_{K}\right)^{-1} \mathbf{Z}_{K}^{\prime}\right) \mathbf{Y}=\mathbf{Y} \boldsymbol{\Lambda}
$$


As the cluster membership matrix $\mathbf{Z}_{K}$ only appears in the second (i.e., the K-means) part of the objective function, an algorithm iterating between these equations and the K-means algorithm applied to $\mathbf{Y}$ is proposed. Note that, as $\alpha$ approaches zero, $\mathbf{Y}$ is forced towards $\mathbf{Z}_{K} \mathbf{G}$. Hence, the problem converges to the GROUPALS objective with an alternative constraint. (The extreme case $\alpha=0$ itself yields a trivial solution where $\mathbf{Y}=\mathbf{Z}_{K}\left(\mathbf{Z}_{K}^{\prime} \mathbf{Z}_{K}\right)^{-1 / 2} \mathbf{E}_{k}$ and $\mathbf{G}=\left(\mathbf{z}_{K}^{\prime} \mathbf{Z}_{K}\right)^{-1 / 2} \mathbf{E}_{k}$, with $\mathbf{E}_{k}$ a $K \times k$ matrix consisting of $k$ orthogonal unit vectors). On the other hand, as $\alpha$ approaches one, the K-means part is virtually ignored and the solution will converge to the tandem approach solution where K-means is applied to the MCA solution.

Iodice D'Enza et al. (2014) show that, similar to the CA and MCA case, MCA-K-means yields a so-called trivial solution consisting of a constant vector corresponding to the largest eigenvalue. This trivial solution can be avoided by centering the indicator matrices. Hence, by replacing the $\mathbf{Z}_{j}$ by $\mathbf{M Z}_{j}$ for all $j=1, \ldots, p$, where $\mathbf{M}$ is the $n$ dimensional centering matrix. Using the centered data, it can be shown that solving (18) involves the least-squares approximation of

$$
\left(\begin{array}{cc}
\frac{1}{p} \alpha \mathbf{D}_{z}^{-1 / 2} \mathbf{Z}^{\prime} \mathbf{M} \mathbf{Z} \mathbf{D}_{z}^{-1 / 2} & \frac{1}{\sqrt{p}} \alpha(1-\alpha) \mathbf{D}_{z}^{-1 / 2} \mathbf{Z}^{\prime} \mathbf{M} \mathbf{Z}_{K} \mathbf{D}_{K}^{-1 / 2} \\
\frac{1}{\sqrt{p}} \alpha(1-\alpha) \mathbf{D}_{K}^{-1 / 2} \mathbf{Z}_{K}^{\prime} \mathbf{M} \mathbf{Z} \mathbf{D}_{z}^{-1 / 2} & (1-\alpha) \mathbf{I}_{K}
\end{array}\right) .
$$

Comparing the lower left block (that is, the last $K$ rows and first $\sum_{j=1}^{p} q_{j}$ columns) of this matrix to equation (4), that is, the new cluster correspondence analysis objective, we see that the new method can be seen as a constrained version of MCA K-means, focusing only on the associations between clusters and variables rather than also considering all two-way associations among them.

\section{3 i-FCB}

Iterative factorial clustering of binary variables (i-FCB) was introduced by Iodice D'Enza \& Palumbo (2013). An extension that allows the analysis of categorical rather than binary variables was presented in Iodice D'Enza et al. (2014). The i-FCB approach can be formulated as non-symmetric correspondence analysis (NSCA: Lauro \& D'Ambra, 1984; Kroonenberg \& Lombardo, 1999) where the dependent (reference) variable is the cluster membership indicator and the explanatory variables are the $p$ categorical variables. Hence, the category quantifications predict cluster membership. Furthermore, to predict cluster membership using the explanatory (categorical) data, the clusters should be optimally separated. That is, the weighted mean cluster scores should vary as much as possible. The i-FCB procedure thus considers two objectives: 1) Obtain a 
non-symmetric correspondence analysis solution for the cross-tabulation of the cluster allocation with the categorical variables. 2) Allocate subjects to clusters in such a way that the variance between weighted cluster means is as large as possible.

In our notation, the first objective becomes:

$$
\min _{\mathbf{B}, \mathbf{G}}\left\|\mathbf{Z}_{K}^{\prime} \mathbf{M Z D}_{z}^{-1}-\mathbf{G B}\right\|^{\prime} \|^{2}
$$

s.t. $\mathbf{B}^{\prime} \mathbf{D}_{z} \mathbf{B}=n q \mathbf{I}_{k}$. For fixed $\mathbf{Z}_{K}$ the solution can be obtained by finding the singular value decomposition

$$
\mathbf{D}_{K}^{\frac{1}{2}} \mathbf{Z}_{K}^{\prime} \mathbf{M Z D}_{z}^{-\frac{1}{2}}=\mathbf{U} \boldsymbol{\Lambda}^{1 / 2} \mathbf{V}^{\prime},
$$

and letting

$$
\mathbf{B}=\sqrt{n q} \mathbf{D}_{z}^{-1 / 2} \mathbf{V} \text { and } \mathbf{G}=\mathbf{D}_{K}^{-1 / 2} \mathbf{U} \boldsymbol{\Lambda}^{1 / 2}=\mathbf{Z}_{K}^{\prime} \mathbf{M Z D}_{z}^{-\frac{1}{2}} \mathbf{V} . .
$$

Using (22), the second objective can be formulated as

$$
\max _{\mathbf{Z}_{K}} \phi=\left\|\mathbf{D}_{K}^{1 / 2} \mathbf{G}\right\|^{2}=\left\|\mathbf{D}_{K}^{1 / 2} \mathbf{Z}_{K}^{\prime} \mathbf{M Z D}_{z}^{-\frac{1}{2}} \mathbf{V}\right\|^{2} .
$$

In a similar fashion as the derivations in Section 3, this problem can be shown to be equivalent to the K-means problem:

$$
\min _{\mathbf{Z}_{K}}\left\|\sqrt{n q} \mathbf{D}_{w} \mathbf{M Z B}-\mathbf{Z}_{K} \mathbf{G}\right\|^{2},
$$

where $\mathbf{D}_{w}=\operatorname{diag}\left(\mathbf{D}_{K} \mathbf{Z}_{K} \mathbf{1}\right)$, that is, the elements of $\mathbf{D}_{w}$ indicate for each subject, the size of the cluster to which it belongs.

To solve the i-FCB objectives, the following algorithm is proposed:

1. Generate an initial cluster allocation $\mathbf{Z}_{K}$ (e.g., by randomly assigning subjects to clusters).

2. Use (22) to obtain a category quantification matrix B.

3. Calculate subject coordinates $\mathbf{Y}=\mathbf{D}_{w} \mathbf{Z B}$

4. Apply K-means to $\mathbf{Y}$ to update the cluster allocation matrix $\mathbf{Z}_{K}$ and return to step 2. Repeat until convergence.

Note that the problems consecutively solved in this problem are not, as was the case in our new method, equivalent. That is, the NSCA objective used to calculate $\mathbf{B}$ (and $\mathbf{G}$ ) does not correspond directly to the K-means objective considered for the cluster allocation update. In particular, 
the coordinates/weights for the clusters are orthonormal in the NSCA framework implying the maximization of $\mathbf{G}^{\prime} \mathbf{G}$ whereas the K-means objective can be shown to correspond to $\mathbf{G}^{\prime} \mathbf{D}_{K} \mathbf{G}$. Moreover, in this algorithm, the K-means procedure is not straightforward as $\mathbf{Y}$ depends on $\mathbf{Z}_{K}$ through $\mathbf{D}_{w}$.

\section{Simulation study}

An extensive comparative study of the different dimension reduction and cluster analysis methods does not exist. Hwang et al. (2006) illustrate their MCA K-means method using one empirical data set and compare the results with those obtained using GROUPALS. Iodice D'Enza et al. (2014) apply GROUPALS, MCA K-means, i-FCB and the tandem approach to one empirical dataset and describe the results. Based on these empirical examples it is not possible to draw clear conclusions concerning the methods' performances nor is it possible to relate them to full dimensional clustering. To overcome these limitations, we propose a simulation study. The objectives of our simulation study are: 1) Assess to what extent the different methods are able to retrieve existing cluster structure in the data. 2) Compare the performance of the different methods with respect to each other. 3) Assess the influence of several factors on the performances.

\subsection{Data generating process}

For interval data, generating high dimensional data based on a low dimensional configuration is relatively straightforward (see, e.g., van de Velden \& Takane, 2012; van de Velden \& Bijmolt, 2006). To generate super indicator matrices corresponding to low dimensional MCA solutions is less trivial. We resolve this problem by generating super indicator matrices based on predetermined distributions over the categories. By selecting distributions that assign relatively large probabilities to certain categories and relatively small ones to others, association structure can be controlled for. Moreover, using cluster specific distributions, cluster structure is readily imposed.

We generate the indicator matrices as follows: For each variable, one category is assigned a high probability and the remaining categories are chosen with, equal, low probabilities. To achieve sufficient structure, we choose the high probability categories to be 4 times as likely as the low probability categories. That is, the high and low probabilities are, respectively, $4 /(4+q-1)$ and $1 /(4+q-1)$, where $q$ denotes the number of categories. For each variable, this pattern, in random order, is used as distribution from which to draw the zero/one observations and these distributions are cluster specific. Hence, all draws from individuals in the same cluster have the same underlying 
distribution. Noise variables can be generated using a distribution with equal probabilities for all categories.

\subsection{Experimental design}

In generating the synthetic data we vary several factor that might effect the performance of the methods. We chose these factors and levels in such a way that "typical" high dimensional categorical data are generated. The following factors and levels considered in the simulation study are: Number of variables. We consider either 5, 10 or 20 variables. Number of categories per variable. We fix the number of categories per variable to 2,5 or 10 categories and also consider a scenario in which, for each variable, we randomly select the number of categories to be either 2, 5 or 10 . Noise: Presence/Absence of noise variables. For the scenarios with noise, we add, respectively, 2, 4 or 8 noise variables to the 5, 10 and 20 variables scenarios. Cluster size distribution: Two cases are considered: Equal sized cluster (balanced) versus unequal sized (unbalanced) cluster sizes. For the unbalanced scenario, the relative cluster sizes are randomly drawn.

For each scenario we simulate 50 data sets of 1000 observations. We analyze each data set by the following methods: Full dimensional clustering, the tandem approach, GROUPALS, MCA K-means, i-FCB and our new cluster correspondence analysis method. We only consider four cluster solutions and, in the (joint) dimension reduction methods, three dimensional solutions. For the full dimensional clustering we use Gower's coefficient for dissimilarity (Gower, 1971) and K-medoids clustering. That is, points are allocated to the closest, in terms of the Gower distance, most common observed pattern in a cluster. To avoid local minima due to the K-means/medoids step, we use 100 random starts for all methods.

\subsection{Evaluation criteria and analysis}

The simulation study allows us to impose cluster structure and hence gauge how well the methods are able to retrieve the underlying clusters. As the "true" cluster structure is known, we are able to compare the obtained cluster solutions with the true cluster allocation. For this purpose, we use the adjusted Rand index (ARI) of Hubert \& Arabie (1985). The ARI assesses the similarity between two cluster solutions, adjusted for chance correspondences between these solutions. The upper limit of the ARI is one, and indicates perfect agreement. An ARI of zero indicates that the method does not improve on random assignment, with all positive values indicating an improvement. Negative ARI values indicate poorer performance than random assignment. 
In practice, the true clustering is unknown. To assess the quality of cluster solutions, several socalled internal cluster validity measures exist. Of these measures, we consider the average silhouette width (Rousseeuw, 1987)). The silhouette width for a point $i$ allocated to cluster $c$, is defined as the average distance of point $i$ with points in the nearest cluster not equal to $c$, say $a_{i c}$, minus the average distance of point $i$ with the other points in cluster $c, b_{i c}$. This differences is normalized by dividing it through the larger of these two average distances. Hence, $s_{i c}=\left(a_{i c}-b_{i c}\right) / \max \left(a_{i c}, b_{i c}\right)$. By definition, the silhouette takes on values between -1 and 1 . Higher values indicate a better separation between the clusters. Negative values are an indication of overlapping clusters. For a fair assessment and comparison of our results, the silhouette widths are calculated using Gower's coefficient for dissimilarity (Gower, 1971) on the original (full dimensional) categorical data.

\subsection{Results}

The cluster retrieval results for the balanced (i.e., the true clusters all have the same size) data can be found in Table 1. We see that by increasing the number of variables and categories, the joint dimension reduction and cluster analysis methods perform better than full dimensional clustering. Adding noise to the data amplifies this result as the reduced dimension methods appear to be unaffected by this. For few (5) variables, i-FCB and the new method have more difficulty in retrieving the true clusters, however, when 10 or more variables are used, all methods perform similarly. The results for the unbalanced scenario, Table 2, are comparable. All methods have more difficulty in retrieving the true clusters than in the balanced case. However, with the exception of i-FCB, which appears to suffer more from the unbalancedness, the differences are small.

Table 3 gives the results for mixed number of categories. We see that, the ARI values for the mixed cases are close to the average of the non-mixed scenarios.

The average silhouette values for the different scenarios are presented in Tables 4 through 6 . We see that, in general, values are close to zero indicating not well separated clusters. The influence of the number of categories on the average silhouette width is rather large and consistent for all methods, with few categories yielding much better results. Although adding noise did not appear to affect cluster allocation for the reduced dimension methods much, it does lead to a drop in the silhouette values for all methods. Apparently, individuals are correctly classified even though the clusters are less clearly separated. 


\begin{tabular}{|c|c|c|c|c|c|c|c|c|c|}
\hline \multicolumn{10}{|c|}{ Balanced } \\
\hline \multicolumn{10}{|l|}{ no noise } \\
\hline & \multicolumn{3}{|c|}{$p=5$} & \multicolumn{3}{|c|}{$p=10$} & \multicolumn{3}{|c|}{$p=20$} \\
\hline Method & $q_{j}=2$ & $q_{j}=5$ & $q_{j}=10$ & $q_{j}=2$ & $q_{j}=5$ & $q_{j}=10$ & $q_{j}=2$ & $q_{j}=5$ & $q_{j}=10$ \\
\hline Full dim. clust. & .35 & .33 & .19 & .60 & .53 & .25 & .86 & .69 & .34 \\
\hline Tandem & .28 & .31 & .17 & .58 & .59 & .39 & .87 & .86 & .68 \\
\hline MCA K-means & .28 & .30 & .17 & .57 & .59 & .39 & .85 & .83 & .66 \\
\hline $\mathrm{i}-\mathrm{FCB}$ & .22 & .12 & .05 & .58 & .59 & .40 & .87 & .86 & .68 \\
\hline GROUPALS & .29 & .23 & .17 & .58 & .59 & .40 & .87 & .86 & .68 \\
\hline Cluster CA & .26 & .13 & .05 & .57 & .59 & .38 & .87 & .86 & .68 \\
\hline \multicolumn{10}{|l|}{ noise } \\
\hline & \multicolumn{3}{|c|}{$p=5$} & \multicolumn{3}{|c|}{$p=10$} & \multicolumn{3}{|c|}{$p=20$} \\
\hline Method & $q_{j}=2$ & $q_{j}=5$ & $q_{j}=10$ & $q_{j}=2$ & $q_{j}=5$ & $q_{j}=10$ & $q_{j}=2$ & $q_{j}=5$ & $q_{j}=10$ \\
\hline Full dim. clust. & .23 & .25 & .14 & .43 & .41 & .19 & .72 & .57 & .27 \\
\hline Tandem & .25 & .28 & .13 & .55 & .58 & .35 & .86 & .85 & .65 \\
\hline MCA K-means & .25 & .28 & .13 & .54 & .57 & .35 & .84 & .82 & .65 \\
\hline i-FCB & .25 & .12 & .05 & .59 & .59 & .37 & .87 & .86 & .66 \\
\hline GROUPALS & .27 & .22 & .14 & .59 & .58 & .37 & .87 & .85 & .66 \\
\hline Cluster CA & .25 & .13 & .05 & .57 & .58 & .35 & .87 & .85 & .65 \\
\hline
\end{tabular}

Table 1: Average Adjusted Rand index (ARI) for simulated data using four equal sized clusters. The considered factors are: number of variables $(5,10,20)$; number of categories per variable $(2,5,10)$; presence/absence of noise variables.

\begin{tabular}{|c|c|c|c|c|c|c|c|c|c|}
\hline \multicolumn{10}{|c|}{ Unbalanced } \\
\hline \multicolumn{10}{|l|}{ no noise } \\
\hline & \multicolumn{3}{|c|}{$p=5$} & \multicolumn{3}{|c|}{$p=10$} & \multicolumn{3}{|c|}{$p=20$} \\
\hline Method & $q_{j}=2$ & $q_{j}=5$ & $q_{j}=10$ & $q_{j}=2$ & $q_{j}=5$ & $q_{j}=10$ & $q_{j}=2$ & $q_{j}=5$ & $q_{j}=10$ \\
\hline Full dim. clust. & .34 & .39 & .27 & .60 & .48 & .25 & .89 & .51 & .28 \\
\hline Tandem & .25 & .26 & .15 & .48 & .49 & .28 & .81 & .82 & .53 \\
\hline MCA K-means & .25 & .24 & .15 & .46 & .45 & .28 & .74 & .64 & .47 \\
\hline $\mathrm{i}-\mathrm{FCB}$ & .19 & .13 & .04 & .38 & .37 & .24 & .59 & .56 & .40 \\
\hline GROUPALS & .25 & .18 & .13 & .46 & .50 & .29 & .80 & .82 & .54 \\
\hline Cluster CA & .24 & .15 & .06 & .45 & .47 & .30 & .78 & .82 & .52 \\
\hline \multicolumn{10}{|l|}{ noise } \\
\hline & \multicolumn{3}{|c|}{$p=5$} & \multicolumn{3}{|c|}{$p=10$} & \multicolumn{3}{|c|}{$p=20$} \\
\hline Method & $q_{j}=2$ & $q_{j}=5$ & $q_{j}=10$ & $q_{j}=2$ & $q_{j}=5$ & $q_{j}=10$ & $q_{j}=2$ & $q_{j}=5$ & $q_{j}=10$ \\
\hline Full dim. clust. & .21 & .22 & .20 & .34 & .34 & .19 & .50 & .43 & .21 \\
\hline Tandem & .24 & .23 & .12 & .44 & .47 & .25 & .77 & .79 & .47 \\
\hline MCA K-means & .23 & .23 & .12 & .42 & .44 & .24 & .72 & .65 & .44 \\
\hline $\mathrm{i}-\mathrm{FCB}$ & .20 & .13 & .04 & .39 & .36 & .21 & .56 & .55 & .37 \\
\hline GROUPALS & .22 & .19 & .13 & .44 & .48 & .25 & .78 & .80 & .48 \\
\hline Cluster CA & .21 & .15 & .06 & .45 & .48 & .23 & .77 & .78 & .45 \\
\hline
\end{tabular}

Table 2: Average Adjusted Rand index (ARI) for simulated data using clusters with different sizes. The considered factors are: number of variables $(5,10,20)$; number of categories per variable $(2,5,10)$; presence/absence of noise variables.

\subsection{Conclusions of the simulation study}

The simulation study shows that dimension reduction improves clustering of high dimensional categorical data. There is no clear winner among the joint methods and the tandem approach also performs quite well. Note that, in our simulation study, the true dimensionality was not controlled for explicitly. Moreover, we did not consider scenarios involving so-called masking variables, that is, variables that "hide" cluster structure in the first dimensions. For categorical data, it is not trivial how to generate such data in a fair and general way. 


\begin{tabular}{|l|c|c|c|c|c|c|}
\hline & \multicolumn{7}{|c|}{ no noise } \\
\hline & \multicolumn{5}{|c|}{ Balanced } & \multicolumn{3}{c|}{ Unalanced } \\
\hline Method & $p=5$ & $p=10$ & $p=20$ & $p=5$ & $p=10$ & $p=20$ \\
\hline Full dim. clust. & .29 & .50 & .69 & .34 & .50 & .50 \\
Tandem & .25 & .51 & .81 & .22 & .41 & .74 \\
MCA K-means & .25 & .50 & .81 & .22 & .39 & .65 \\
i-FCB & .11 & .52 & .82 & .13 & .32 & .52 \\
GROUPALS & .16 & .52 & .81 & .13 & .41 & .74 \\
Cluster CA & .10 & .50 & .81 & .12 & .42 & .72 \\
\hline \hline & \multicolumn{7}{|c|}{ noise } \\
\hline & $p=5$ & $p=10$ & $p=20$ & $p=5$ & $p=10$ & $p=20$ \\
\hline Method & .22 & .37 & .55 & .21 & .30 & .40 \\
\hline Full dim. clust. & .23 & .49 & .80 & .19 & .37 & .69 \\
Tandem & .22 & .49 & .79 & .19 & .36 & .62 \\
MCA K-means & .11 & .51 & .81 & .10 & .31 & .51 \\
i-FCB & .16 & .50 & .80 & .14 & .39 & .70 \\
GROUPALS & .11 & .49 & .81 & .12 & .42 & .69 \\
Cluster CA & \multicolumn{7}{|c|}{} \\
\hline \hline
\end{tabular}

Table 3: Average Adjusted Rand index (ARI) for simulated data. The considered factors are: balanced groups and unbalanced groups ; presence/absence of noise variables.; number of variables $(5,10,20)$ and a mixed distribution of categories per variable.

\begin{tabular}{|c|c|c|c|c|c|c|c|c|c|}
\hline \multicolumn{10}{|c|}{ Balanced } \\
\hline \multicolumn{10}{|l|}{ no noise } \\
\hline & \multicolumn{3}{|c|}{$p=5$} & \multicolumn{3}{|c|}{$p=10$} & \multicolumn{3}{|c|}{$p=20$} \\
\hline Method & $p_{j}=2$ & $p_{j}=5$ & $p_{j}=10$ & $p_{j}=2$ & $p_{j}=5$ & $p_{j}=10$ & $p_{j}=2$ & $p_{j}=5$ & $p_{j}=10$ \\
\hline Full dim. clust. & .40 & .14 & .06 & .29 & .11 & .04 & .28 & .10 & .03 \\
\hline Tandem & .39 & .14 & .06 & .29 & .12 & .05 & .28 & .11 & .04 \\
\hline MCA K-means & .39 & .14 & .06 & .29 & .12 & .05 & .28 & .11 & .04 \\
\hline i-FCB & .40 & .20 & .10 & .29 & .12 & .05 & .28 & .11 & .04 \\
\hline GROUPALS & .41 & .17 & .07 & .29 & .12 & .05 & .28 & .11 & .04 \\
\hline Cluster CA & .41 & .20 & .09 & .29 & .12 & .05 & .28 & .11 & .04 \\
\hline \multicolumn{10}{|l|}{ noise } \\
\hline & \multicolumn{3}{|c|}{$p=5$} & \multicolumn{3}{|c|}{$p=10$} & \multicolumn{3}{|c|}{$p=20$} \\
\hline Method & $p_{j}=2$ & $p_{j}=5$ & $p_{j}=10$ & $p_{j}=2$ & $p_{j}=5$ & $p_{j}=10$ & $p_{j}=2$ & $p_{j}=5$ & $p_{j}=10$ \\
\hline Full dim. clust. & .24 & .08 & .04 & .17 & .06 & .02 & .18 & .06 & .01 \\
\hline Tandem & .25 & .09 & .04 & .19 & .08 & .03 & .20 & .08 & .03 \\
\hline MCA K-means & .25 & .09 & .04 & .19 & .08 & .03 & .19 & .07 & .03 \\
\hline i-FCB & .27 & .14 & .07 & .20 & .08 & .03 & .20 & .08 & .03 \\
\hline GROUPALS & .27 & .12 & .05 & .20 & .08 & .03 & .20 & .08 & .03 \\
\hline Cluster CA & .27 & .14 & .06 & .20 & .08 & .03 & .20 & .08 & .03 \\
\hline
\end{tabular}

Table 4: Average silhouette index for simulated data using four equal sized clusters. The considered factors are: number of variables $(5,10,20)$; number of categories per variable $(2,5,10)$; presence/absence of noise variables. 


\begin{tabular}{|c|c|c|c|c|c|c|c|c|c|}
\hline \multicolumn{10}{|c|}{ Unbalanced } \\
\hline \multicolumn{10}{|l|}{ no noise } \\
\hline & \multicolumn{3}{|c|}{$p=5$} & \multicolumn{3}{|c|}{$p=10$} & \multicolumn{3}{|c|}{$p=20$} \\
\hline Method & $p_{j}=2$ & $p_{j}=5$ & $p_{j}=10$ & $p_{j}=2$ & $p_{j}=5$ & $p_{j}=10$ & $p_{j}=2$ & $p_{j}=5$ & $p_{j}=10$ \\
\hline Full dim. clust. & .43 & .14 & .06 & .28 & .09 & .03 & .28 & .06 & .02 \\
\hline Tandem & .40 & .13 & .06 & .27 & .10 & .04 & .27 & .11 & .03 \\
\hline MCA K-means & .40 & .12 & .06 & .26 & .10 & .04 & .24 & .08 & .03 \\
\hline i-FCB & .40 & .20 & .10 & .26 & .10 & .04 & .21 & .08 & .03 \\
\hline GROUPALS & .41 & .18 & .07 & .27 & .11 & .04 & .27 & .11 & .04 \\
\hline Cluster CA & .42 & .20 & .09 & .27 & .11 & .04 & .26 & .11 & .04 \\
\hline \multicolumn{10}{|l|}{ noise } \\
\hline & \multicolumn{3}{|c|}{$p=5$} & \multicolumn{3}{|c|}{$p=10$} & \multicolumn{3}{|c|}{$p=20$} \\
\hline Method & $p_{j}=2$ & $p_{j}=5$ & $p_{j}=10$ & $p_{j}=2$ & $p_{j}=5$ & $p_{j}=10$ & $p_{j}=2$ & $p_{j}=5$ & $p_{j}=10$ \\
\hline Full dim. clust. & .25 & .07 & .04 & .15 & .05 & .02 & .12 & .04 & .01 \\
\hline Tandem & .25 & .09 & .04 & .17 & .07 & .03 & .18 & .07 & .02 \\
\hline MCA K-means & .25 & .09 & .04 & .16 & .07 & .03 & .16 & .06 & .02 \\
\hline i-FCB & .27 & .14 & .07 & .17 & .07 & .03 & .14 & .06 & .02 \\
\hline GROUPALS & .27 & .12 & .05 & .18 & .07 & .03 & .18 & .07 & .02 \\
\hline Cluster CA & .28 & .14 & .07 & .18 & .08 & .03 & .18 & .07 & .02 \\
\hline
\end{tabular}

Table 5: Average silhouette index for simulated data using clusters with different sizes. The considered factors are: number of variables $(5,10,20)$; number of categories per variable $(2,5,10)$; presence/absence of noise variables.

\begin{tabular}{|l|c|c|c|c|c|c|}
\hline & \multicolumn{7}{|c|}{ no noise } \\
\hline & \multicolumn{3}{|c|}{ Balanced } & \multicolumn{3}{c|}{ Unalanced } \\
\hline Method & $p=5$ & $p=10$ & $p=20$ & $p=5$ & $p=10$ & $p=20$ \\
\hline Full dim. clust. & .16 & .11 & .10 & .18 & .10 & .06 \\
\hline Tandem & .14 & .11 & .11 & .14 & .09 & .10 \\
\hline MCA K-means & .14 & .10 & .11 & .14 & .08 & .09 \\
\hline i-FCB & .21 & .11 & .11 & .21 & .09 & .08 \\
\hline GROUPALS & .17 & .11 & .11 & .19 & .09 & .10 \\
\hline Cluster CA & .19 & .11 & .11 & .20 & .10 & .10 \\
\hline & \multicolumn{7}{|c|}{ noise } \\
\hline & $p=5$ & $p=10$ & $p=20$ & $p=5$ & $p=10$ & $p=20$ \\
\hline Method & .10 & .07 & .06 & .11 & .05 & .04 \\
\hline Full dim. clust. & .09 & .07 & .08 & .09 & .06 & .07 \\
\hline Tandem & .09 & .07 & .08 & .09 & .06 & .06 \\
\hline MCA K-means & .15 & .08 & .08 & .15 & .06 & .05 \\
\hline i-FCB & .12 & .08 & .08 & .13 & .06 & .07 \\
\hline GROUPALS & .14 & .08 & .08 & .14 & .07 & .07 \\
\hline Cluster CA & \multicolumn{7}{|c|}{ Unalanced } \\
\hline
\end{tabular}

Table 6: Average silhouette index for simulated data. The considered factors are: balanced groups (top of the table) and unbalanced groups (bottom of the table); presence/absence of noise variables.; number of variables $(5,10,20)$ and a mixed distribution of categories per variable. 


\section{Application}

We apply our method to the results of a personality test, the Humor Styles Questionnaire, proposed by Martin et al. (2003). This questionnaire has been developed to measure four independent ways in which people express and appreciate humor: affiliative, defined as the benign uses of humor to enhance one's relationships with others; self-enhancing, indicating uses of humor to enhance the self; aggressive, the use of humor to enhance the self at the expense of others; self-defeating the use of humor to enhance relationships at the expense of oneself. The questionnaire consists of 32 statements rated from 1 to 5 according to the respondents' level of agreement. The number of respondents is $n=993$. The 32 statements and corresponding labels are reported in Table 7 .

Martin et al. (2003) used the questionnaire to construct the humor styles. Here, we analyze the data from a different perspective: Can we distinguish clusters of individuals with similar humor profiles? We apply the new cluster CA method to the data and use a two dimensional, three cluster solution. The solution depicting clusters and attributes is displayed in Figure 1. Using equation (12) we can project individual subject points into this CA map and thus visualize the variability within and between clusters. Figure 2 gives the corresponding map.

In $\mathrm{CA}$, the origin depicts the average profile and all other points depict deviations from this average profile. The two dimensional displays, depicts two clearly separated clusters and one central cluster. To interpret the solution we consider individual attributes (i.e., a statement and category combination) and the positions of the cluster mean points relative to these. Note that, in cluster CA, the solotion for cluster means and category quantifications constitute a biplot. Hence, these projections can be used to retrieve the observed values (see also Greenacre, 1993, on the biplot interpretation of correspondence analysis, in particular, on how to reconstruct the original data entries from a biplot).

From the two dimensional plot it is clear that cluster 1 appears to be associated with extreme categories (i.e., $1 \mathrm{~s}$ or $5 \mathrm{~s}$ ) for the statements concerning self-defeating humor and self-enhancing humor. People in this group use humor to deal with bad situations (self-enhancing humor) and do so at their own expense. On the other side of the spectrum we find a cluster of individuals (cluster 3 in Figure 1) indicating a preference for affiliative humor. They show disagreement on statements concerning not laughing with others (and, agreement on "laughing with close friends"). The individuals in this cluster also indicate more than average disagreement concerning the statements regarding the use of humor to enhance the self. Furthermore, individuals in this cluster do not appreciate self-defeating humor. Finally, the cluster closest to the center of the plot (i.e., cluster 


\begin{tabular}{|c|c|c|c|}
\hline Label & Statement & Humor style & $\begin{array}{l}\text { Original } \\
\text { question code }\end{array}$ \\
\hline AF1 & I usually don't laugh or joke around much with other people. & Affiliative humor & Q1 \\
\hline AF2 & $\begin{array}{l}\text { I don't have to work very hard at making other people laugh, } \\
\text { I seem to be a naturally humorous person. }\end{array}$ & Affiliative humor & Q5 \\
\hline AF3 & I rarely make other people laugh by telling funny stories about myself. & Affiliative humor & Q9 \\
\hline AF4 & I laugh and joke a lot with my closest friends. & Affiliative humor & Q13 \\
\hline AF5 & I usually don't like to tell jokes or amuse people. & Affiliative humor & Q17 \\
\hline AF6 & I enjoy making people laugh. & Affiliative humor & Q21 \\
\hline AF7 & I don't often joke around with my friends. & Affiliative humor & Q25 \\
\hline AF8 & I usually can't think of witty things to say when I'm with other people. & Affiliative humor & Q29 \\
\hline SE1 & If I am feeling depressed, I can usually cheer myself up with humor. & Self-enhancing humor & Q2 \\
\hline SE2 & Even when I'm by myself, I'm often amused by the absurdities of life. & Self-enhancing humor & Q6 \\
\hline SE3 & $\begin{array}{l}\text { If I am feeling upset or unhappy, I usually try to think of something } \\
\text { funny about the situation to make myself feel better. }\end{array}$ & Self-enhancing humor & Q10 \\
\hline SE4 & $\begin{array}{l}\text { My humorous outlook on life keeps me from } \\
\text { getting overly upset or depressed about things. }\end{array}$ & Self-enhancing humor & Q14 \\
\hline SE5 & $\begin{array}{l}\text { If I'm by myself and I'm feeling unhappy, I make an effort to think of } \\
\text { something funny to cheer myself up. }\end{array}$ & Self-enhancing humor & Q18 \\
\hline SE6 & If I am feeling sad or upset, I usually lose my sense of humor. & Self-enhancing humor & Q22 \\
\hline SE7 & $\begin{array}{l}\text { It is my experience that thinking about some amusing aspect } \\
\text { of a situation is often a very effective way of coping with problems. }\end{array}$ & Self-enhancing humor & Q26 \\
\hline SE8 & $\begin{array}{l}\text { I don't need to be with other people to feel amused } \\
\text { I can usually find things to laugh about even when I'm by myself. }\end{array}$ & Self-enhancing humor & Q30 \\
\hline AG1 & If someone makes a mistake, I will often tease them about it. & Aggressive humor & Q3 \\
\hline AG2 & People are never offended or hurt by my sense of humor. & Aggressive humor & Q7 \\
\hline AG3 & $\begin{array}{l}\text { When telling jokes or saying funny things, I am usually not very } \\
\text { concerned about how other people are taking it. }\end{array}$ & Aggressive humor & Q11 \\
\hline AG4 & $\begin{array}{l}\text { I do not like it when people use humor as a way } \\
\text { of criticizing or putting someone down. }\end{array}$ & Aggressive humor & Q15 \\
\hline AG5 & $\begin{array}{l}\text { Sometimes I think of something that is so funny } \\
\text { that I can't stop myself from saying it, } \\
\text { even if it is not appropriate for the situation. }\end{array}$ & Aggressive humor & Q19 \\
\hline AG6 & I never participate in laughing at others even if all my friends are doing it. & Aggressive humor & Q23 \\
\hline AG7 & If I don't like someone, I often use humor or teasing to put them down. & Aggressive humor & Q27 \\
\hline AG8 & $\begin{array}{l}\text { Even if something is really funny to me, } \\
\text { I will not laugh or joke about it if someone will be offended. }\end{array}$ & Aggressive humor & Q31 \\
\hline SD1 & I let people laugh at me or make fun at my expense more than I should. & Self-defeating humor & Q4 \\
\hline SD2 & $\begin{array}{l}\text { I will often get carried away in putting myself down } \\
\text { if it makes my family or friends laugh. }\end{array}$ & Self-defeating humor & Q8 \\
\hline SD3 & $\begin{array}{l}\text { I often try to make people like or accept me more by saying something } \\
\text { funny about my own weaknesses, blunders, or faults. }\end{array}$ & Self-defeating humor & Q12 \\
\hline SD4 & I don't often say funny things to put myself down. & Self-defeating humor & Q16 \\
\hline SD5 & $\begin{array}{l}\text { I often go overboard in putting myself down when } \\
\text { I am making jokes or trying to be funny. }\end{array}$ & Self-defeating humor & Q20 \\
\hline SD6 & $\begin{array}{l}\text { When I am with friends or family, I often seem to be the one that } \\
\text { other people make fun of or joke about. }\end{array}$ & Self-defeating humor & Q24 \\
\hline SD7 & $\begin{array}{l}\text { If I am having problems or feeling unhappy, } \\
\text { I often cover it up by joking around, } \\
\text { so that even my closest friends don't know how I really feel. }\end{array}$ & Self-defeating humor & Q28 \\
\hline SD8 & $\begin{array}{l}\text { Letting others laugh at me is my way of keeping } \\
\text { my friends and family in good spirits. }\end{array}$ & Self-defeating humor & Q32 \\
\hline
\end{tabular}

Table 7: Humor Styles Questionnaire: Each statement is rated from 1 (strongly disagree), to 5 (strongly agree); for each statement, the corresponding humor style and original question number is reported

2 in Figure 1) does not show extreme agreement/disagreement concerning any statement. People in this cluster exhibit preferences that are closely aligned with the average preferences. For these data this corresponds to agreement levels close to the center of the scale for most statements.

The interpretation given above is based on the visualization in Figure 1. To help with the interpretation of clusters it is useful to identify attributes that deviate the most from the independence condition. The three plots on the left side of Figure 3 (i.e. 3a, 3c, and 3e) show for each cluster the twenty attributes with the highest standardized residuals (positive or negative). A positive (negative) residual means that the attribute has an above (below) average frequency within the 
cluster. Figure 3 clearly confirms the graphical depiction of Figure 1 . We see that for cluster 1, agreement is high for the statements concerning the self-defeating and self-enhancing humor styles. (Note that some items indicate disagreement, however those items, for example SD4, are phrased reversely). Cluster 3 is characterized by respondents with an affiliative humor style, as the group is mostly characterized by strong agreement on sentences (AF1, AF5, AF7, AF4, AF6, AF8), with AF1 and AF4 being on a reverse scale. This group also indicates disagreement with several of the self-defeating and self-enhancing humor styles. Finally, in cluster 2, respondents are less pronounced in their levels of agreement with the various humor styles. Instead, they tend to show medium levels of agreement on many attributes.

We compare the results of cluster CA with those of the other methods described in the paper. A true clustering is not known so we can only consider similarity of the low dimensional configurations and the different cluster partitions. Concerning the similarity of the configurations, Table 9 gives the congruency coefficients (Borg \& Groenen, 2005, pp. 437-440) between the attribute configurations. We see that the cluster CA solution is similar to the configuration obtained using GROUPALS. Also, similarity with the Tandem approach (i.e., the two dimensional MCA solution) is high. For these data, it appears that MCA K-means yields a less similar configuration.

To compare the different cluster partitions we use the adjusted Rand index (ARI). We consider the results of all methods including full dimensional clustering where, as before in the simulation study, we use Gower dissimilarities and K-medoids clustering. The results are in Table 10. Again we see that the cluster CA solution is similar to the GROUPALS solution (.88), and, to a lesser degree, the tandem and MCA K-means solutions (.84 and .83, respectively). Both full dimensional clustering and i-FCB yield rather different cluster partitions. Full dimensional clustering in particular yields a solution that is quite different with ARI values around .18 for all comparisons. These differences are also apparent when comparing the cluster size distributions in Table 8 .

Similarity and dissimilarity of the methods with respect to each other does not indicate which method is better. However, based on the simulation study, the joint dimension methods are expected to perform better than the full dimensional clustering solution. This expectation is confirmed when considering the average silhouette width. Rounded to two decimals, this is for all dimension reduction and clustering methods .07 whereas the value for full dimensional clustering equals .03.

Such findings are also evident from the two dimensional maps in Figure 1. In Figure 3, the 20 largest (in absolute value) standardized residuals per attribute are depicted for the three clusters obtained using cluster CA (the three figures on the left) and full dimensional clustering (the three 
figures on the right). The clusters of the full dimensional solution have been ordered in such a way that they match the cluster size order of cluster CA. This side by side comparison, clearly illustrates that the clusters obtained using the new method are easier to interpret than those obtained using the full dimensional cluster results.

\begin{tabular}{rrrr}
\hline & 1 & 2 & 3 \\
\hline fullDim & .27 & .43 & .30 \\
Tandem & .18 & .45 & .37 \\
MCAk & .18 & .46 & .36 \\
iFCB & .27 & .38 & .35 \\
Groupals & .18 & .45 & .37 \\
CAclus & .18 & .43 & .39 \\
\hline
\end{tabular}

Table 8: Relative cluster size distributions: clusters are ordered to match the Cluster CA solution order

\begin{tabular}{lcccc}
\hline & Tandem & MCA K-means & i-FCB & GROUPALS \\
\hline Tandem & & & & \\
MCA K-means & .78 & & & \\
i-FCB & .95 & .77 & & \\
GROUPALS & .97 & .79 & .95 & \\
Cluster CA & .86 & .68 & .90 & .90 \\
\hline
\end{tabular}

Table 9: Two-by-two congruency index of the low-dimensional attribute configurations as produced by the methods

\begin{tabular}{lccccc}
\hline & Full dim. clust. & Tandem & MCA K-means & i-FCB & GROUPALS \\
\hline Full dim. clust. & & & & & \\
Tandem & .17 & & & & \\
MCA K-means & .17 & .95 & & & \\
i-FCB & .16 & .60 & .58 & & \\
GROUPALS & .17 & .91 & .91 & .57 & .88 \\
Cluster CA & .18 & .84 & .83 & .59 & .89 \\
\hline
\end{tabular}

Table 10: Adjusted Rand indices between the different partitions

\section{Conclusions}

This paper proposes a new method that combines cluster analysis and correspondence analysis. The new method can be seen as correspondence analysis of a cluster by variable association table and yields, in addition to a low-dimensional approximation depicting clusters and attributes, a cluster partitioning of individuals based on the profiles over the categorical variables. We showed how the new method relates to existing methods for joint dimension reduction and clustering of categorical data. Using a simulation study, we assessed the performances of the methods. Upon the 


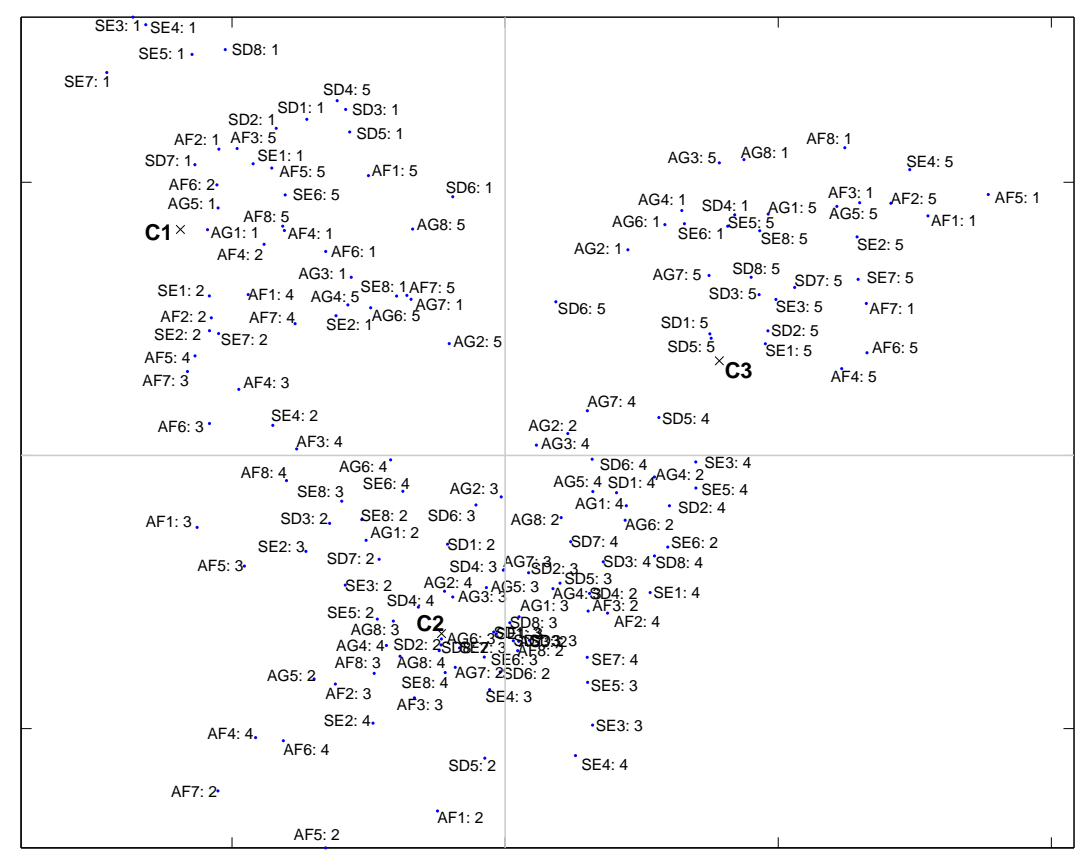

Figure 1: Cluster Correspondence analysis biplot. Scaling as defined in equation (14). Attribute labels correspond to the labels in Table 7 with category numbers added. Cluster means are labelled C1 through C3.

results of our simulation study we can state that categorical data clustering benefits from dimension reduction. That is, with respect to retrieval of true underlying cluster structure, joint dimension reduction and clustering methods outperform full dimensional clustering for high dimensional.

Among the joint dimension reduction and clustering methods, differences were relatively small both with respect to cluster retrieval and internal cluster validity. This is not surprising because data coding and centering were the same for all the considered methods. However, there are some important points in favor of the new method. First, when cluster sizes are not equal, the i-FCB method has an higher failure rate than the other methods. Secondly, although it is possible in MCA K-means to obtain and plot individual subject points, the coordinates of these subject points are not insightful as they are influenced by the (user selected) weights assigned to the MCA and K-means part of the objective. With respect to these weights it should be noted that, in this paper, we only considered equal weights. It is not clear which criteria to use to tune this parameter but results are dependent on that choice.

For continuous (interval) data, Vichi \& Kiers (2001) showed that a so-called tandem approach 


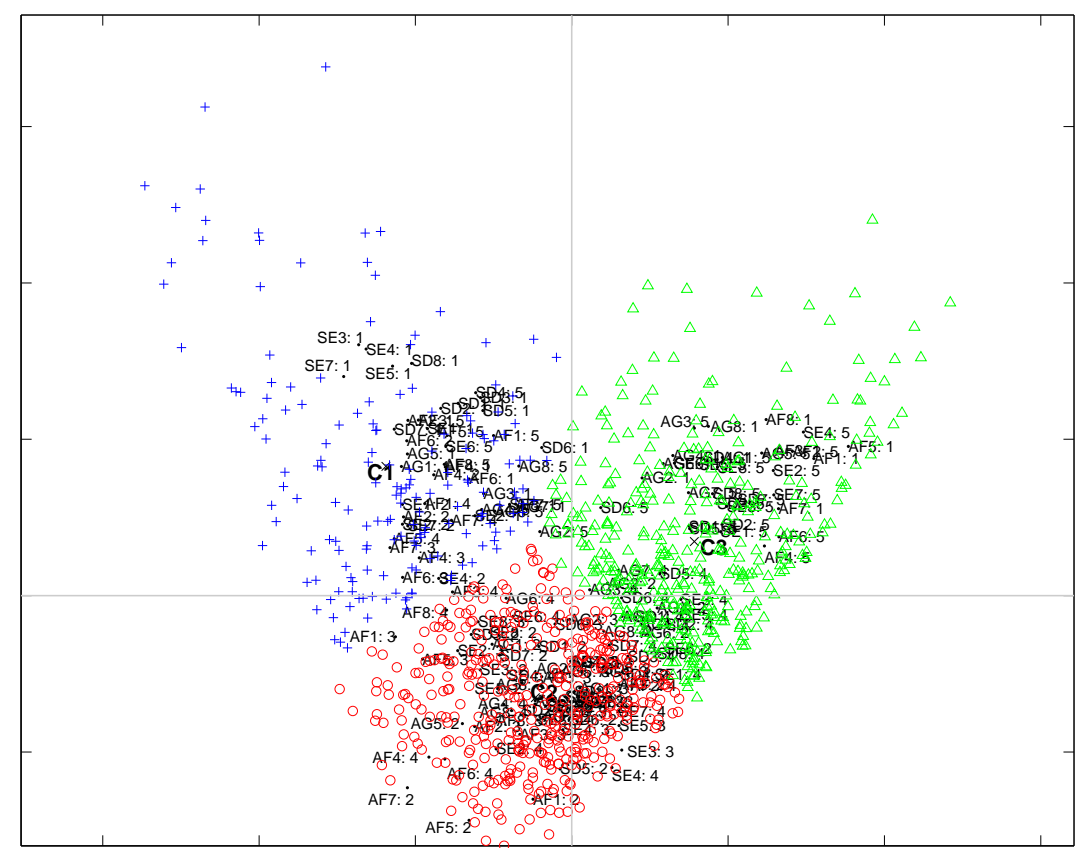

Figure 2: Cluster Correspondence analysis biplot with projected subject points. Scaling as defined in equation (14). Attribute labels correspond to the labels in Table 7 with category numbers added. Cluster 1 points are represented by ' + ', cluster 2 points by ' $\circ$ ' and cluster 3 points by ' $\triangle$ '.

in which clustering is performed after dimension reduction, could be problematic. In our simulation study, we did not find evidence for this in the categorical variable case. Unlike the simulation study designed by Vichi \& Kiers (2001), we did not consider scenarios in which so-called masking variables were used to hide cluster structure in the reduced space. It could be the case that scenarios can be constructed were the tandem approach does suffer from the sequential analysis.

Our simulation clearly demonstrated that for high dimensional categorical data dimension reduction improves the clustering results. In presence of noise variables (i.e., variables unrelated to the cluster structure) the difference increased. Possible reasons for this failure of full dimensional clustering versus joint dimension reduction approaches, are the fact that the true dimensionality of the data is typically not equal to the size of the data table and that the exaggerated dimensionality of the data table contains noise that is filtered out in the joint dimension reduction methods. 


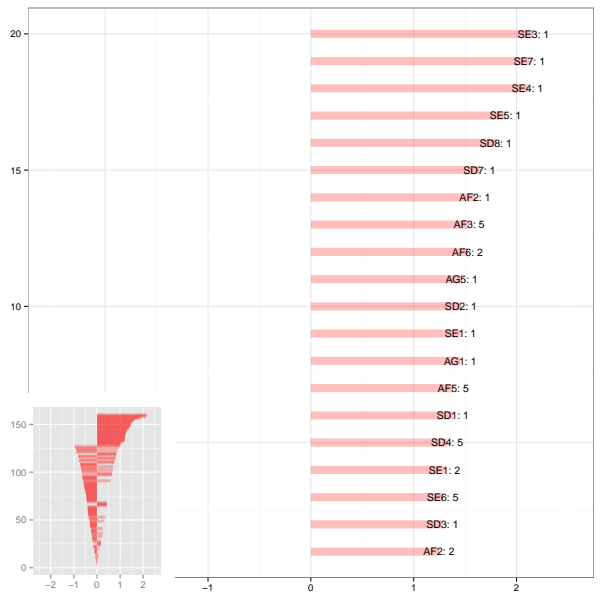

(a) Cluster CA: Cluster 1

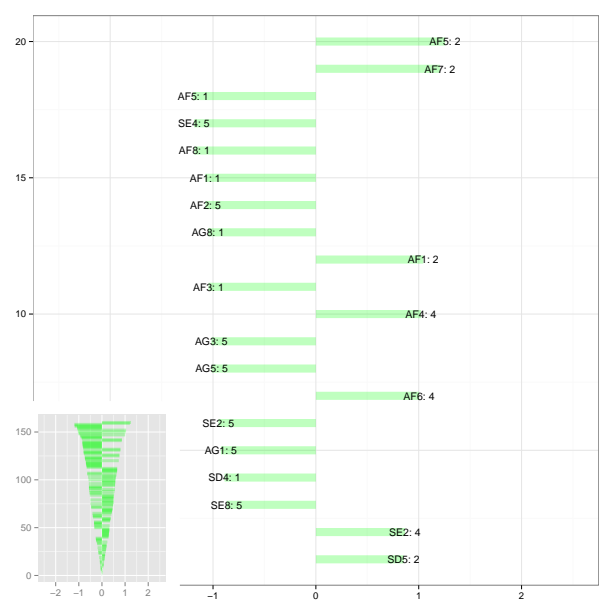

(c) Cluster CA: Cluster 2

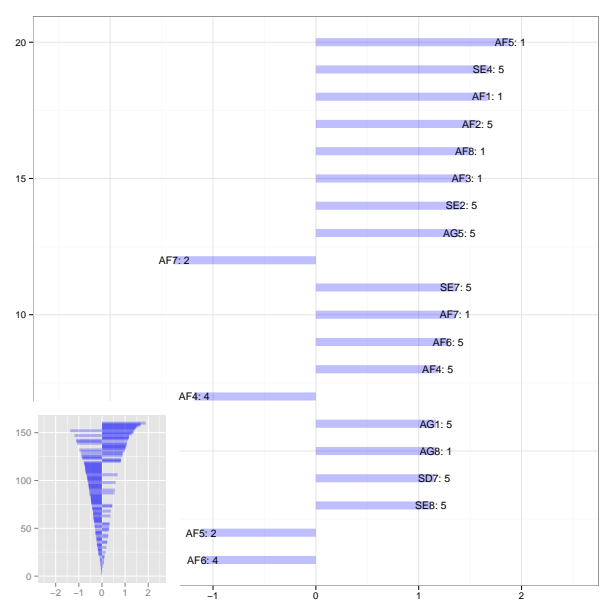

(e) Cluster CA: Cluster 3

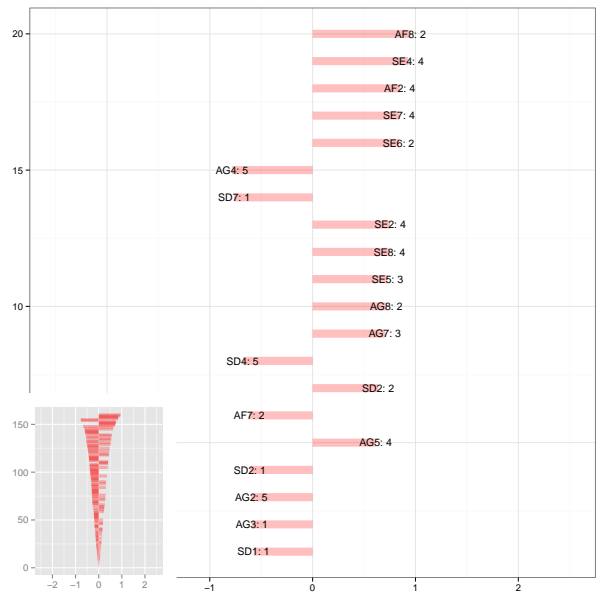

(b) Full dim. cluster: Cluster 1

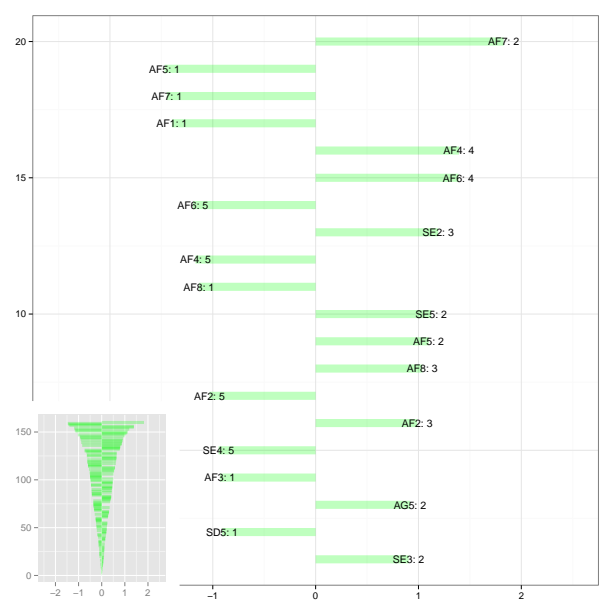

(d) Full dim. cluster: Cluster 2

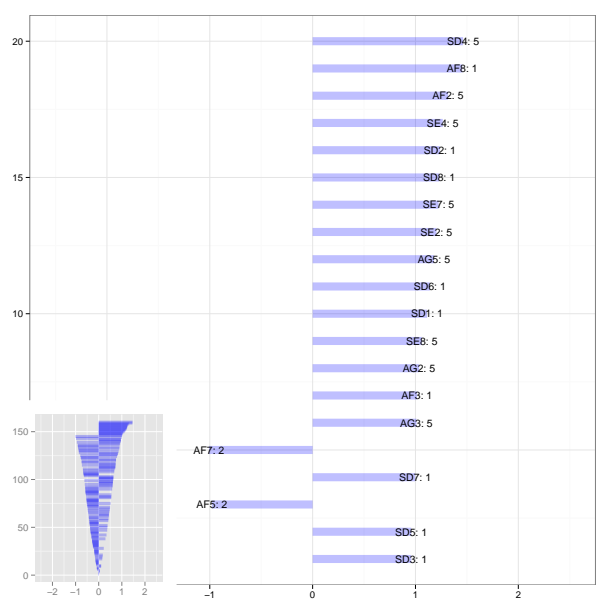

(f) Full dim. cluster: Cluster 3

Figure 3: Top 20's of the largest standardized residuals per cluster (with complete distributions in small subplots) for Cluster CA (left) and full dimensional clustering (right) 


\section{References}

Borg, I., \& Groenen, P. J. (2005). Modern multidimensional scaling: Theory and applications. Springer.

De Soete, G., \& Carroll, J. D. (1994). K-means clustering in a low-dimensional euclidean space. In E. Diday, Y. Lechevallier, M. Schader, P. Bertrand, \& B. Burtschy (Eds.), New approaches in classification and data analysis (p. 212-219). Springer-Verlag, Berlin.

Gower, J. C. (1971). A general coefficient of similarity and some of its properties. Biometrics, 27, 623-637.

Gower, J. C., Gardner Lubbe, S., \& Le Roux, N. J. (2011). Understanding biplots. John Wiley \& Sons.

Gower, J. C., Groenen, P. J. F., \& van de Velden, M. (2010). Area biplots. Journal of Computational and Graphical Statistics, 19(1), 46-61.

Gower, J. C., \& Hand, D. J. (1996). Biplots. London: Chapman and Hall.

Greenacre, M. J. (1984). Theory and applications of correspondence analysis. London: Academic Press.

Greenacre, M. J. (1993). Biplots in correspondence analysis. Journal of Applied Statistics, 20(2), $251-269$.

Hubert, L., \& Arabie, P. (1985). Comparing partitions. Journal of Classification, 2(1), 193-218. Retrieved from http://dx.doi.org/10.1007/BF01908075 doi: 10.1007/BF01908075

Hwang, H., Dillon, W. R., \& Takane, Y. (2006). An extension of multiple correspondence analysis for identifying heterogenous subgroups of respondents. Psychometrika, 71, 161-171.

Iodice D’Enza, A., \& Palumbo, F. (2013). Iterative factor clustering of binary data. Computational Statistics, 789-807. Retrieved from http://dx.doi.org/10.1007/s00180-012-0329-x $(10.1007 / \mathrm{s} 00180-012-0329-\mathrm{x})$

Iodice D’Enza, A., van de Velden, M., \& Palumbo, F. (2014). On joint dimension reduction and clustering of categorical data. In D. Vicari, A. Okada, G. Ragozini, \& C. Weihs (Eds.), Analysis and modeling of complex data in behavioral and social sciences. Springer, Berlin. 
Kroonenberg, P. M., \& Lombardo, R. (1999). Nonsymmetric correspondence analysis: a tool for analysing contingency tables with a dependence structure. Multivariate Behavioral Research, $34,367-396$.

Lauro, N., \& D’Ambra, L. (1984). L' analyse non symetrique des correspondances [nonsymmetric correspondence analysis]. In E. Diday, L. Lebart, M. Jambu, \& Thomassone (Eds.), Data analysis and informatics iii (p. 433-446). Elsevier, Amsterdam.

Martin, R. A., Puhlik-Doris, P., Larsen, G., Gray, J., \& Weir, K. (2003). Individual differences in uses of humor and their relation to psychological well-being: Development of the humor styles questionnaire. Journal of research in personality, 37(1), 48-75.

Nishisato, S. (1980). Analysis of categorical data: dual scaling and its applications. Toronto: University of Toronto Press.

Nishisato, S. (1994). Elements of dual scaling: an introduction to practical data analysis. Hillsdale, New Jersey: Lawrence Erlbaum Associates.

Rousseeuw, P. J. (1987). Silhouettes: A graphical aid to the interpretation and validation of cluster analysis. Journal of Computational and Applied Mathematics, 20(0), 53 - 65. Retrieved from http://www.sciencedirect.com/science/article/pii/0377042787901257 doi: http://dx .doi.org/10.1016/0377-0427(87)90125-7

van de Velden, M., \& Bijmolt, T. (2006). Generalized canonical correlation analysis of matrices with missing rows: a simulation study. Psychometrika, 71 (2), 323-331.

van de Velden, M., \& Takane, Y. (2012). Generalized canonical correlation analysis with missing values. Computational Statistics, $27(3), 551-571$.

Van Buuren, S., \& Heiser, W. (1989). Clustering n objects into k groups under optimal scaling of variables. Psychometrika, 54, 699-706.

Vichi, M., \& Kiers, H. A. L. (2001). Factorial k-means analysis for two-way data. Computational Statistics and Data Analysis, 37, 49-64.

Vichi, M., Vicari, D., \& Kiers, H. (2009). Clustering and dimensional reduction for mixed variables. (Unpublished manuscript)

Yamamoto, M., \& Hwang, H. (2014). A general formulation of cluster analysis with dimension reduction and subspace separation. Behaviormetrika, 41, 115-129. 